This item was submitted to Loughborough's Research Repository by the author.

Items in Figshare are protected by copyright, with all rights reserved, unless otherwise indicated.

\title{
Model-based fault detection and control design - applied to a pneumatic industrial application
}

PLEASE CITE THE PUBLISHED VERSION

https://engineering.purdue.edu/IFACMech10/

PUBLISHER

(C) IFAC

VERSION

AM (Accepted Manuscript)

LICENCE

CC BY-NC-ND 4.0

\section{REPOSITORY RECORD}

Grewal, Karmjit Singh, Roger Dixon, Roger M. Goodall, and John T. Pearson. 2019. "Model-based Fault Detection and Control Design - Applied to a Pneumatic Industrial Application". figshare. https://hdl.handle.net/2134/6604. 
This item was submitted to Loughborough's Institutional Repository (https://dspace.lboro.ac.uk/) by the author and is made available under the following Creative Commons Licence conditions.

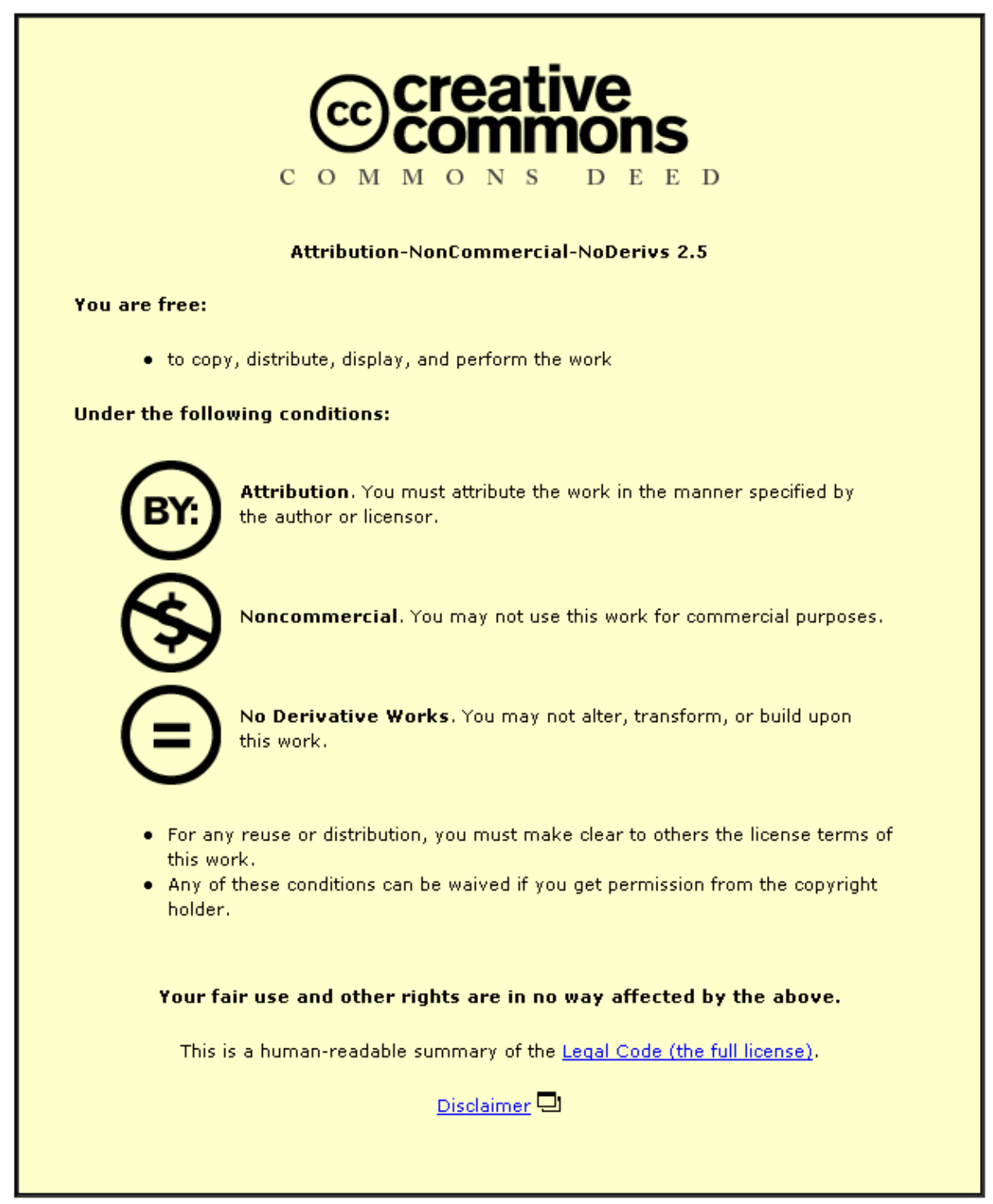

For the full text of this licence, please go to: http://creativecommons.org/licenses/by-nc-nd/2.5/ 


\title{
Model-based fault detection and control design - Applied to a pneumatic industrial application
}

\author{
K.S.Grewal $^{\mathrm{a}^{*}}$, R. Dixon ${ }^{\mathrm{a}}$, R. Goodall ${ }^{\mathrm{a}}$, J. Pearson ${ }^{\mathrm{b}}$ \\ ${ }^{a}$ Control Systems Group, Loughborough University. Loughborough. Leicestershire. UK. (Email:k.grewal@lboro.ac.uk) \\ ${ }^{b}$ SEIC, BAE Systems, Holywell Park, Loughborough. Leicestershire. UK. (email: j.t.pearson@iee.org)
}

\begin{abstract}
In safety critical applications it is important to detect faults that may compromise system safety and to take appropriate action. This paper discusses research carried-out on the development and validation of a model-based fault detection and isolation (FDI) system for a pneumatically actuated Stewart platform. The FDI scheme is based on combining parityequation and Kalman filter based techniques. The parity and Kalman filter equations are formulated and used to generate residuals that, in turn, are analysed to determine whether faults are present in the system. Details of the design process are given and the experimental results are compared. The results demonstrate that both approaches when combined can successfully detect and isolate and in some cases accommodate faults associated with the sensors, actuators (servo-valves and piping) and the pneumatic system itself.
\end{abstract}

Keywords: Fault detection; isolation; residuals; modelling; pneumatic; parity equation; Kalman filter; Stewart-Gough platform.

\section{INTRODUCTION}

A traditional approach to fault tolerance is based on hardware or physical redundancy methods to use multiple sensors, actuators, components to measure a particular variable. One of the major problems inherited with hardware redundancy includes the extra equipment and maintenance costs (Isermann, 1997). However, in certain safety critical applications physical redundancy can sometimes be the only method available.

Model-based Fault Detection and Isolation (FDI) uses the principles of analytical redundancy to first detect deviations from normal behaviour in a system, and then to isolate the particular component that has a fault. There are a number of approaches to model-based residual generation. Useful surveys of these and other useful FDI methods can be found in Patton (1997), Isermann (1984), Frank, (1990), Willsky (1976), and Venkatasubramaniam et al (2003).

Fig.1 depicts a schematic structure of a typical FDI procedure using analytical redundancy described by Isermann, (1984). The analytical approach requires that a residual generator perform a validation of the nominal relationships of the system, using the actual input, $\mathbf{u}$, and the measured output, y). Information from normal system operation (no faults, normal system behaviour) must be received, in order to act as a benchmark for comparison.

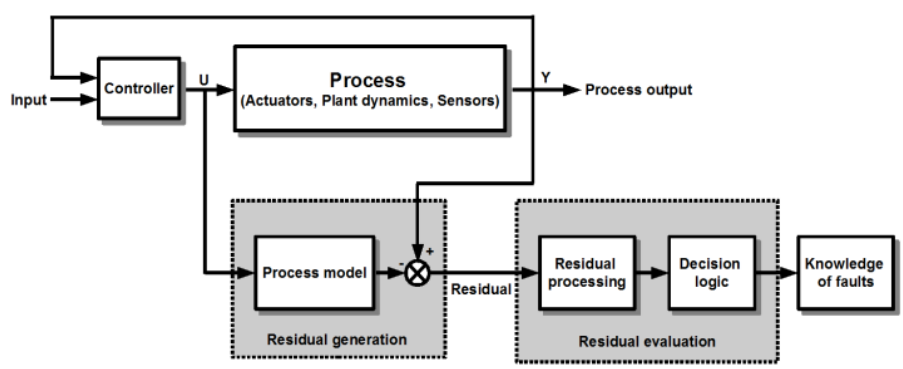

Fig.1: Schematic structure of a FDI procedure using analytical redundancy.
This is usually achieved by expressing the normal system operation in terms of system models. This modelling procedure is necessary to have relationships between various known or measured physical variables. Such system models can be represented in different formats (quantitative or qualitative).

This paper describes the design, development and validation of a fault tolerant control system on a StewartGough platform comprising six pneumatic actuators. The results show that the FDI scheme is found to detect and isolate pneumatic and sensor faults and in some cases an accommodation action is applied. As far as the authors are aware no such work has been carried out on a pneumatic system.

\section{EXPERIMENTAL SET-UP}

Stewart-Gough platforms are generally of a mechanical design used mainly for position control. The design is a parallel mechanism consisting of a rigid body mobile plate, connected to a fixed base plate and is defined by at least three stationary points on the fixed (grounded) base connected to six independent legs. The six legs are connected to both the base and top plate by universal/ball joints in parallel located at both ends of the six legs. The legs are designed with an upper body and lower body that can be adjusted. This allows for the length of each leg to be varied. The linear extension and retraction of the six cylinders gives the platform six degrees of freedom positioning capabilities. The aim is to control the pneumatic actuators (legs) using position control, with the platform position (6-degrees of freedom) achieved by a supervisory controller. Figure 2 shows the StewartGough platform set-up. The design concept of the FDI scheme for the full Stewart-Gough platform is first designed using a single cylinder set-up. This modular approach is adopted so that a robust fault tolerant control scheme can be designed and validated off-line (i.e. not attached to the Stewart-Gough platform). This modular approach is made 
possible as the Stewart-Gough platform design uses six identical pneumatic cylinders. The single actuator set-up is illustrated schematically in Fig.3. The flow from the air supply is governed by the servo valve which gives a flow into each of the cylinder chambers that is proportional to the voltage applied. This results in a differential pressure across the cylinder piston causing it to move. The pressures are measured via pressure sensors located between the valve and cylinder chambers (as close to the cylinder chambers as possible). The overall aim is position control, so this is measured via two Linear Resistive Transducers (LRT) mounted in the cylinder. The second position sensor provides a means of redundancy if the primary position develops a fault or fails. The schematic also shows the xPC Target coupled with Matlab/Simulink, which provides a real-time environment for running the control and fault detection algorithms.

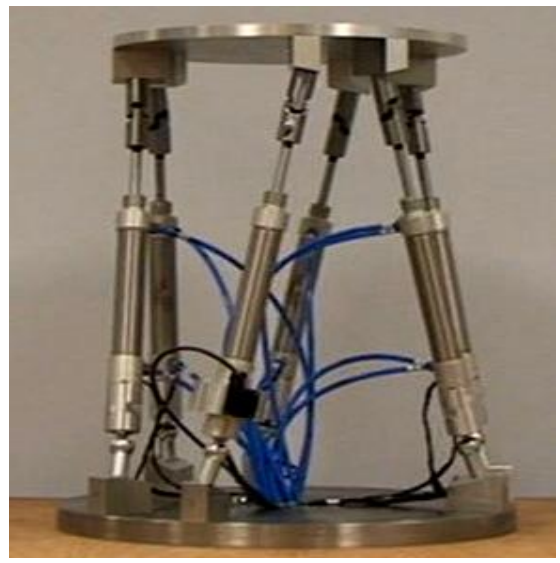

Fig.2. Stewart-Gough platform set-up

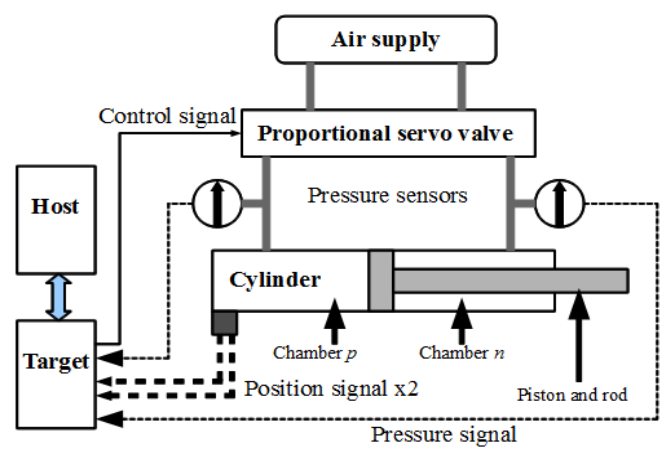

Fig.3. Schematic of experimental set-up

\section{MODELLING PNEUMATIC SYSTEM}

The relationship between the air mass flow and the pressure changes in the chambers is obtained using energy conservation laws (first law of thermodynamics), and the force equilibrium is given by Newton's second law. The pneumatic system can be approximated by equations (1-3), see for example Grewal et al (2008). Where $P_{p}$ is the pressure in chamber $p, P_{n}$ is the pressure in chamber $n, V_{p}$ is the air volume in chamber $p, V_{n}$ is the air volume in chamber in $n, T_{s}$ is the operating temperature, $\gamma$ is the ratio of specific heat, and $R$ is the universal gas constant. $M$ is the piston mass, $A$ is the bore area, $x$ is the position of the piston, and $F_{f}$ represents the viscous friction coefficient and coulomb friction force. $K$ is the servo valve constant $\left(\mathrm{kg} \cdot \mathrm{s}^{-1} \cdot \mathrm{V}^{-1}\right)$ determined from the valve's data-sheet and $v$ is the voltage input.

$$
\begin{aligned}
& \dot{P}_{p}=-\frac{\gamma A P_{p}}{V_{p}} \dot{x}+K \frac{\gamma R T_{s}}{V_{p}} v \\
& \dot{P}_{n}=\frac{\gamma A P_{n}}{V_{n}} \dot{x}-K \frac{\gamma R T_{s}}{V_{n}} v \\
& \ddot{x}=\frac{A}{M}\left(P_{p}-P_{n}\right)-\frac{F_{f}}{M} \Delta \dot{x}
\end{aligned}
$$

\section{DESIGN OF FDI SCHEME}

Figure 1 shows the generic structure of the model-based fault detection scheme. The method consists of detecting faults on the process, which includes actuators, components and sensors, based on measuring the input signal $U(t)$ and the output signal $Y(t)$. The detection method uses models to generate residuals $R(t)$. The residual evaluation examines the residuals for the likelihood of faults and a decision rule is applied to determine if faults have occurred. Referring to the pneumatic system depicted in Fig.3, the proportional valve would be described as the actuator and the pneumatic cylinder would be described as the plant. The sensors are self-evident. In this paper the process model can be based on either parity equations or Kalman filters. The main function of the FDI scheme is to detect faults such as position/pressure sensor faults, leaks in pneumatic system, blockages in pneumatic system and actuator faults. Once these faults are detected the FDI scheme isolates the fault. In isolating the fault, the fault can be classified as a particular fault. The two approaches taken here using the parity equation and Kalman filter technique are discussed, in turn, below.

\subsection{The Parity Equation Method}

The basic idea is to provide a proper check of the parity (consistency) of the measurements for the monitored system. The idea of the parity approach is to rearrange the model structure to achieve the best fault isolation (i.e. so that the effect of faults is far greater than that of the other uncertainties). The desired properties for the residual signal are $R(t) \neq 0$ if $f(t) \neq 0$. Where $R$ is the residual and $f$ is the fault. The residual is generated based on the information provided by the system input and output signals and the plant equation. Fig.4 shows the pneumatic control loop scheme, which contains the following elements: The controller $C(s)$, the proportional valve $G A(s)$, the pneumatic actuator $G P(s)$, and the sensor $G S(s)$. The proportional valve fault $F a(s)$ and the sensor fault $F S(s)$ can have dynamics, which are modelled by the transfer functions $H a(s)$, and $H S(s)$. In addition to the position (feedback) sensor, pressure sensors are included in the system to read pressure from each chamber of the actuator. These are not included in the closed loop system, and are shown as $P p(s)$ and $P n(s)$ respectively. With the pressure sensor faults, shown as $F P p(s)$ and $F P n(s)$, again having dynamics modelled by the transfer functions $H P p(s)$ and $H P n(s)$. Using the description of the system shown in 
Fig.4 the following relationships (equations) can be derived. (Grewal et al, 2009).

$X S(s)=[G S(s)+H S(s) F S(s)][G A(s) U(s) G P(s)+H a(s) F a(s) G A(s)$

$G P(s)]$

$P n_{a c t}=[U(s) G A(s)+H a(s) F a(s)][P n(s)+H P n(s) F P n(s)]$

$P p_{a c t}=[U(s) G A(s)+H a(s) F a(s)][P p(s)+H P p(s) F P p(s)]$

$U(s)=C(s)(V(s)-X S(s))$

With the current experimental set-up the pneumatic plant output can only be measured with the position sensor. Therefore the actuator and plant faults cannot be isolated. Residuals are formulated from (4) to (6) as follows

$R_{l}=X S(s)-G S(s) G P(s) G A(s) U(s)=H S(s) F S(s)+H a(s) F a(s)$

$R_{2}=P n_{a c t}-U(s) G A(s) P n(s)=H a(s) F a(s)+H P n(s) F P n(\mathrm{~s})$

$R_{3}=P p_{a c t}-U(s) G A(s) P p(s)=H a(s) F a(s)+H P p(s) F P p(\mathrm{~s})$

To represent the pneumatic process shown in Fig.4, $G A(s)$ is modelled by the equations (1) and (2) and $G P(s)$ by equation (3). It is assumed that the fault and sensor transfer functions are all instantaneous i.e. $\mathrm{Ha}(s), H S(s), H P n(s)$, $H P p(s), P n(s), P p(s)$ and $G S(s)=1$.

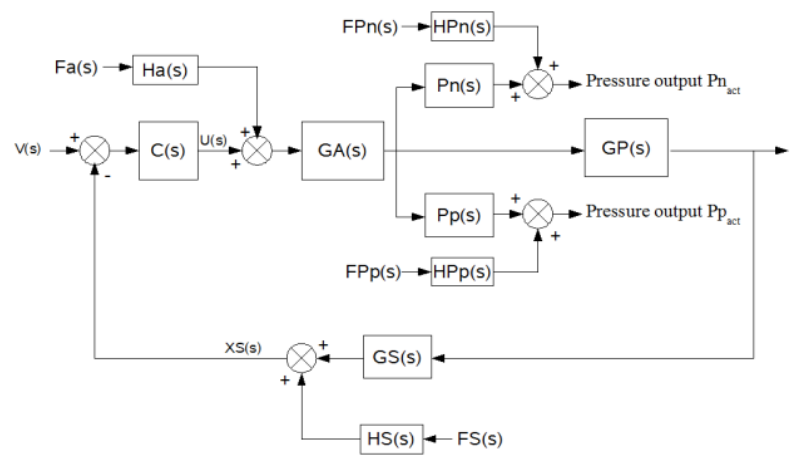

Fig.4. Pneumatic closed loop scheme with intended faults

\subsection{Observer approach (Kalman filter)}

The basic idea of the observer approach is to reconstruct the outputs of the system from the measurements or subsets of measurements with the aid of observers or Kalman filters using the estimation error or innovation (Frank, 1990). This estimation error or innovation is used as a residual for the detection and isolation of faults. For a linear system:

$\dot{x}=A x+B u+G w \quad$ (State equations)

$y=C x+D u+H w+v \quad$ (Measurement equations)

Where $u$ is the input, $w$ is the process noise, $v$ is the measurement noise with $E\left(w w^{T}\right)=Q$, and $E\left(v v^{T}\right)=R$. It is also assumed that the state and measurement noise is uncorrelated, that is, $E\left(w v^{T}\right)=0$. An optimal estimate of $y$, $\hat{y}$ can be provided by the Kalman filter equations:

$\dot{\hat{x}}=A \hat{x}+B u+L(y-C \hat{x}-D u)$ and

$$
\hat{y}=C \hat{x}+D u
$$

Where in practice the weightings for process and measurement noise ( $\mathrm{Q}$ and $\mathrm{R}$ respectively) are chosen heuristically using engineering judgement to provide a tradeoff between sensitivity to faults, and the likelihood of false alarms. The steady-state Kalman filter gain $L$ is determined by solving an algebraic Riccati equation. This estimator uses the known inputs $u$ and the measurement $y$ to generate the output and state estimates $\hat{y}$ and $\hat{x}$. In order to make the system model equations observable, equations (1-3) have been manipulated to achieve system observability (15), the model has been reduced from a $4^{\text {th }}$ order to a $3^{\text {rd }}$ order equation.

$$
\begin{aligned}
& {\left[\begin{array}{c}
\dot{P}_{d} \\
\dot{x} \\
\ddot{x}
\end{array}\right]=\left[\begin{array}{ccc}
0 & 0 & a-b \\
0 & 0 & 1 \\
\frac{A}{M} & 0 & \frac{-F_{f}}{M}
\end{array}\right]\left[\begin{array}{c}
P_{d} \\
x \\
\dot{x}
\end{array}\right]+\left[\begin{array}{c}
c-d \\
0 \\
0
\end{array}\right]} \\
& y_{p o s}=\left[\begin{array}{lll}
0 & 1 & 0
\end{array}\right]\left[\begin{array}{c}
P_{d} \\
x \\
\dot{x}
\end{array}\right] \\
& y_{p_{d}}=\left[\begin{array}{lll}
1 & 0 & 0
\end{array}\right]\left[\begin{array}{c}
P_{d} \\
x \\
\dot{x}
\end{array}\right]
\end{aligned}
$$

Where

$P_{d}=P_{p}-P_{n}, a=-\frac{\gamma A P_{p}}{V_{p}}, b=\frac{\gamma A P_{n}}{V_{n}}, c=K \frac{\gamma R T_{s}}{V_{p}}, d=-K \frac{\gamma R T_{s}}{V_{n}}$

In designing the Kalman filter approach only the sensed outputs are considered. These are position and pressure difference outputs. The residual equations are:

$$
\begin{aligned}
& R_{4}=y_{p o s}-C_{p o s} \hat{x}_{1} \\
& R_{5}=y_{P_{d}}-C_{P_{d}} \hat{x}_{2}
\end{aligned}
$$

Where

$$
C_{p o s}=\left[\begin{array}{lll}
0 & 1 & 0
\end{array}\right], C_{P_{d}}=\left[\begin{array}{lll}
1 & 0 & 0
\end{array}\right]
$$

\subsection{Redundant sensor voting scheme}

The voter scheme is used to minimize switching transients since the isolation of faulty signals is achieved through a continuous numerical weighting (Broen, 1975). The voter scheme continuously determines the output in a manner which discriminates against the erroneous signal in favour of the other channels. The general form (Fig.5) of the voter scheme is determined using a weighted average of its inputs.

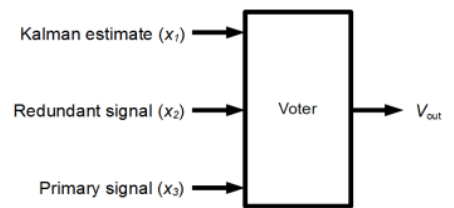

Fig.5. General form of voting scheme

Where $V_{\text {out }}$ is defined as

$$
V_{\text {out }}=\frac{w_{1} x_{1}+w_{2} x_{2}+w_{3} x_{3}}{w_{1}+w_{2}+w_{3}}
$$


The numerical properties of the voting scheme is given by letting

$$
\widehat{w_{j}}=\frac{w_{j}}{w_{1}+w_{2}+w_{3}}, \quad j=1,2, \text { and } 3
$$

Where $w_{j}$ is given by

$$
\begin{aligned}
& w_{1}=\left[1+\left(\frac{x_{1}-x_{2}}{a}\right)^{2}\left(\frac{x_{1}-x_{2}}{a}\right)^{2}\right]^{-1} \\
& w_{2}=\left[1+\left(\frac{x_{2}-x_{1}}{a}\right)^{2}\left(\frac{x_{2}-x_{3}}{a}\right)^{2}\right]^{-1} \\
& w_{3}=\left[1+\left(\frac{x_{3}-x_{1}}{a}\right)^{2}\left(\frac{x_{3}-x_{2}}{a}\right)^{2}\right]^{-1}
\end{aligned}
$$

Where $a$ is the tolerance parameter and is the measure of allowable noise level in a given channel and is hosen heuristically using engineering judgement. It should be noted as the above voting scheme deals with three sensor inputs. The Kalman filter estimate $\left(x_{3}\right)$ and the redundant signal $\left(x_{2}\right)$, the third signal is taken from the primary signal $\left(x_{3}\right)$.

\subsection{Residual Evaluation and Thresholds}

The purpose of residual evaluation is to generate a fault decision by processing the residuals. Residual evaluation is essentially to check if the residual is responding to a fault. The residual evaluation can in its simplest form be a threshold test applied to the residual, i.e. a fault is assumed present if $\left|R_{i}(t)\right|>J_{i}(t)$ where $J_{i}(t)$ is the threshold of the $i^{t h}$ residual. Another method may consist of statistical sequential probability ratio testing. In the present case the residuals are processed to acquire the root mean square (RMS) of the value over a moving window of $N$ samples (Dixon, 2004) as shown:

$$
R_{i R M S}(k)=\sqrt{\frac{\sum_{j=k-N}^{k} R_{i j}{ }^{2}}{N}} \quad i=1,2,3,4,5
$$

Where $R_{i}(k)$ is the value of the $i^{\text {th }}$ residual at the $k^{\text {th }}$ sample. Subsequently, the residual RMS value is compared with a predetermined fault detection threshold. Once a residual exceeds its respective threshold a fault flag is raised, the fault flag remains high as triggering is set so that once the threshold is exceeded the flag remains raised.

Table 1 shows the theoretical fault signatures using the parity equations and Kalman filter approaches of the pneumatic system for various faults. These signatures arise from the formulation of parity equations and the structure of the observer scheme. Where the parity equations residuals $\left(R_{1}, R_{2}\right.$ and $\left.R_{3}\right)$, are given in (8), (9), and (10). The Kalman filter residuals $\left(R_{4}\right.$ and $\left.R_{5}\right)$ are given by equations (15), (16), and (17). From (19), (20), and (21) further residuals can be generated. Basically, if no faults occur the weighted output is 1 and if a fault occurs in either of the three signals $\left(x_{1}, x_{2}\right.$ and $\left.\mathrm{x}_{3}\right)\left(\widehat{w}_{j}\right) \rightarrow 0$. In order to comply with the Kalman and parity residuals the weighted average outputs are inverted (i.e. fault $=1$, and no fault $=0$ ).
Table 1: Residual fault signatures

\begin{tabular}{|ccccccccc|}
\hline Residual & Actuator & Plant & $\left(x_{3}\right)$ & $\left(x_{2}\right)$ & $\left(x_{1}\right)$ & $\begin{array}{c}\text { Pressure } \\
\text { sensor }(\mathrm{p})\end{array}$ & $\begin{array}{c}\text { Pressure } \\
\text { sensor (n) }\end{array}$ \\
\hline$R_{1}$ & 1 & 1 & 1 & 1 & 1 & 0 & 0 \\
\hline$R_{2}$ & 1 & 1 & 0 & 0 & 0 & 1 & 0 \\
\hline$R_{3}$ & 1 & 1 & 0 & 0 & 0 & 0 & 1 \\
\hline$R_{4}$ & 1 & 1 & 1 & 1 & 1 & 0 & 0 \\
\hline$R_{5}$ & 1 & 1 & 0 & 0 & 0 & 1 & 1 \\
\hline$w_{1}$ & 0 & 0 & 0 & 0 & 1 & 0 & 0 \\
\hline$w_{2}$ & 0 & 0 & 0 & 1 & 0 & 0 & 0 \\
\hline$w_{3}$ & 0 & 0 & 1 & 0 & 0 & 0 & 0 \\
\hline
\end{tabular}

\section{EXPERIMENTAL RESULTS}

In order to demonstrate the FDI scheme using parity equations and Kalman filter approaches a number of experiments were carried out on the Stewart-Gough platform (Fig.2). The faults presented are actuator faults including pressure leaks and pipe blockages and position/pressure sensor faults (signal loss and signal drift). These faults occur on just one pneumatic cylinder. The results for the other cylinders will of course be the same. The demand input to the system is a series of motions that represent the 6-degrees of movement, i.e. the longitudinal motions (surge), lateral (sway), and vertical (heave), and the angular motions expressed as Eulerian angle rotations with respect to the $\mathrm{x}$ axis (roll), y-axis (pitch), and z-axis (yaw).

When a fault occurs, appropriate (safe) actions need to be taken. For the position sensor fault, accommodation is possible. This is also the case for the pressure sensor. For the other faults the appropriate action is to shutdown the rig. Actuator and leak faults are discussed in sections 5.1 and 5.2. These results are typical and the full set of results is summarised in Table 2.

\subsection{Actuator fault (control signal loss)}

A fault $\mathrm{Fa}(\mathrm{s})$ (see Fig.4) is applied to the proportional valve. The fault injected is that the control signal has been disconnected. This is physically achieved by means of a switch. Fig.6 shows the time histories of this experiment (actuator fault).

\subsubsection{Discussion - Actuator fault (control signal loss)}

Applying the disconnection fault to the control signal of the proportional valve has an effect on the parity residual $\left(R_{1}\right)$, this raises the fault flag. The fault has an effect on the pressure sensor parity residuals $\left(R_{2}\right.$ and $\left.R_{3}\right)$. Both position and pressure difference Kalman residuals $\left(R_{4}\right.$ and $\left.R_{5}\right)$ are affected by the actuator fault and their fault flags are raised. Residuals $w_{1}, w_{2}$ and $w_{3}$ are not affected and the respective fault flags remain low. This agrees with the fault signatures detailed in Table 1. With this particular fault accommodation is not available as the control signal to the servo valve of pneumatic cylinder 2 is lost. This means that the desired positional movement of the rig is inadequate. From here (21.62s) the safety sequence is activated and the platform is made safe (i.e. brought back to its rest position). From both methods (Kalman and parity) the Kalman approach tracks the fault better and has a faster fault detection response time. 
Overall, it is clear that the parity equations and the Kalman filter approaches can detect an actuator fault. However, neither method can isolate the fault in terms of discriminating between actuator and plant (cylinder) fault.
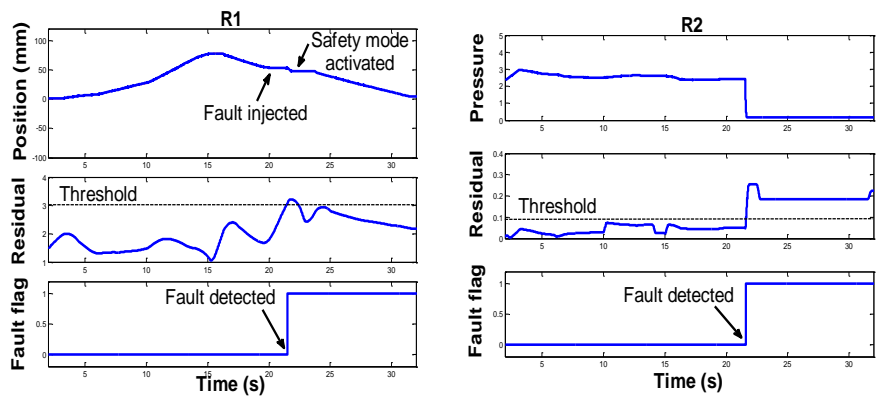

R3
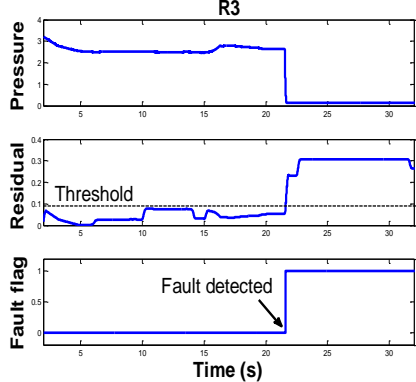

R5
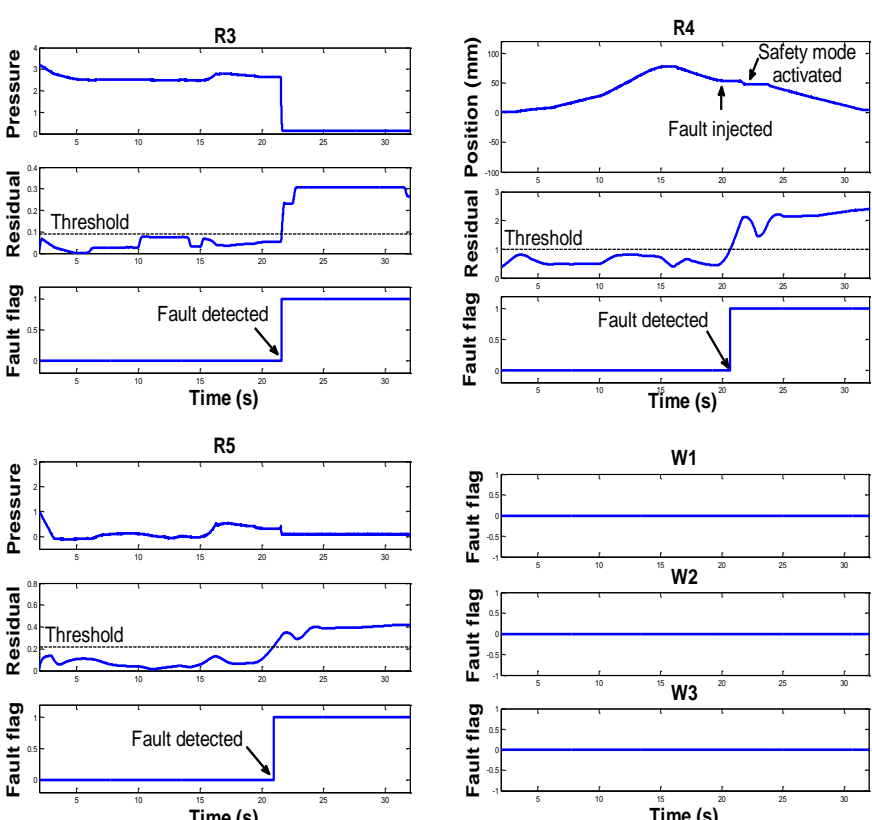

W1
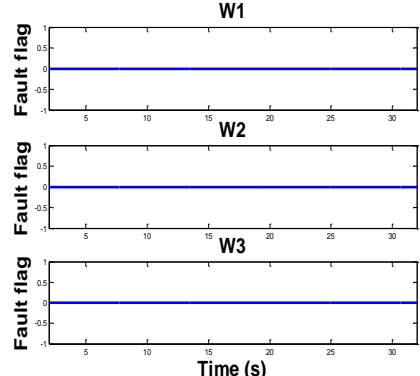

Fig.6. Actuator fault $\mathrm{Fa}(\mathrm{s})$ (control signal loss) results

\subsection{Air leak (between servo valve and pneumatic cylinder)}

Air leaks may occur during normal operating conditions and can appear from any part of the pneumatic system. In this case the leak is injected between the servo valve and pneumatic cylinder at 18.6s. Figure 7 shows the time histories of this experiment (leak fault).

\subsubsection{Discussion-Air leak}

Applying the leak fault to the pneumatic cylinder has an effect on the parity residual $R_{l}$, this raises the fault flag. The fault also has an affect on the pressure sensor parity residuals $\left(R_{2}\right.$ and $\left.R_{3}\right)$. The fault affects the position residual $R_{4}$ and the pressure difference residual $R_{5}$. Residuals $w_{1}, w_{2}$ and $w_{3}$ are not affected and the respective fault flags remain low. This agrees with the fault signatures detailed in Table 1. With this type of fault, accommodation is not available as pressure is lost between the servo valve and pneumatic cylinder. This means that the desired positional movement of the rig is not achievable. However, from the point where the fault is induced (18.6s) and the final residual $\left(R_{l}\right)$ is raised $(23.98 \mathrm{~s})$ a certain amount of fault tolerance is available. Once the final residual fault flag is raised the safety sequence is activated and the platform is made safe (i.e. brought back to its rest position). Comparing RMS residuals $R_{l}$ and $R_{4}$ (position outputs), the Kalman filter approach when compared with the parity equation scheme has a faster fault detection response time.
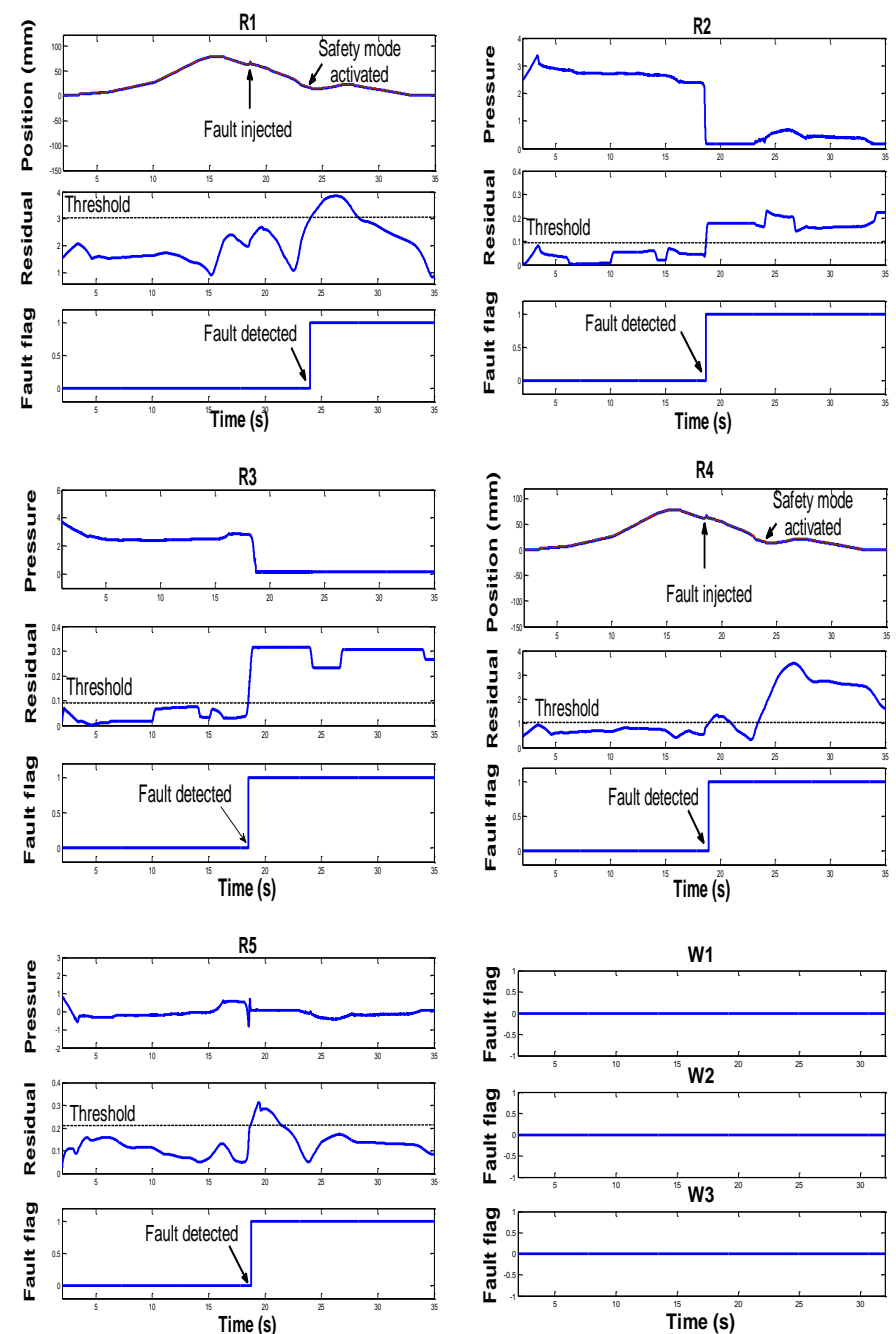

Fig.7. Air leak fault results

\subsection{Summary of fault detection results}

Table 2 shows a summary of the full set of the fault experiments carried out. In terms of comparison between the parity equations and the Kalman filter residuals outputs, the Kalman approach was found to be the more responsive in detecting faults. For the various faults applied Table 2 shows that the residual fault flags concur with the residual fault signatures detailed in Table 1. Furthermore, the results show that when all five residuals $R_{1}, R_{2}, R_{3}, R_{4}$, and $R_{5}$ are raised, the faults can be classified as a pneumatic fault or control signal. Whereas, in the case of the position sensor fault Residuals $R_{1}, R_{4}$ and $w_{3}$ are raised. The pressure sensors faults can be identified as to which sensor is faulty by their respective residuals (i.e. residual's $R_{2}$ and $R_{5}$ for pressure sensor $p$ and residual's $R_{3}$ and $R_{5}$ for pressure sensor $n$. 


\section{CONCLUSIONS}

The paper has described the design, test and evaluation of fault detection in a closed loop system for a pneumatic Stewart-Gough platform. Parity equations and the Kalman filter approach have been used to generate residuals for the purpose of fault detection. Various fault scenarios have been considered, which are typical for a pneumatic operating system. These faults have been applied to the pneumatic system and the results show that using the described parity equation and Kalman filter methods; including the weighted average voting scheme (for position sensor faults) fault detection and isolation was possible from the available measurements. An important reason for selecting the parity equation approach is that it is a relatively simple design approach. Basic equations of the system are used directly and compared to the system. The Kalman filter approach is more complex as the scheme takes into account noise variances and requires tuning. The authors believe that applying the three schemes allows for enhanced fault detection and fault isolation.

\section{REFERNCES}

Broen, R. B. (1975). New voters for redundant systems. ASME Journal of Dynamic Systems, Measurement and Control, pp. 41-45.

Dixon, R (2004). Observer-based FDIA: application to an electromechanical positioning system. Control Engineering Practice 12, pp. 1113-1125.
Frank, P. M. (1990). Fault diagnosis in dynamic systems using analytical and knowledge-based redundancy: A survey and some new results. Auto.26, pp. 459-474.

Grewal, K. S., Dixon, R., and Pearson. J. (2008). Development of a fault tolerant actuation systemmodelling and validation. Actuator 08, $11^{\text {th }}$ international conference on new actuators, pp, 469-472.

Grewal, K.S., Dixon, R. and Pearson, J. (2009). Comparison of two model based residual generation schemes for the purpose of fault detection and isolation applied to a pneumatic actuation system. SafeProcess 2009, Barcelona, Spain, 30 June - 3 July 2009, pp.1420-1425.

Isermann, R. (1984). Process fault detection based on modelling and estimation methods- A survey. Automatica, 20, pp. 387-404.

Isermann, R. (1997). Supervision, Fault-Detection and Fault Diagnosis methods- An Introduction, Control Engineering Practice, 5, (5), pp. 639-652.

Patton, R.J (1997). Fault-tolerant control: The 1997 situation. Proceedings of the third symposium on fault detection, supervision and safety for technical processes (Safeprocess'97). 3, pp, 1029-1052.

Venkatasubramanian, V., Rengaswamy, R., Yin, K., and Kavuri, S.N. (2003). A review of process fault detection and diagnosis. Part I: Quantitative model-based methods. Computers and Chemical Engineering, 27, pp. 293-311.

Willsky, A. S. (1976). A survey of design methods for failure detection in dynamic systems. Automatica, 12, 601-611.

Table 2: Experimental results summary

\begin{tabular}{|c|c|c|c|c|c|c|c|}
\hline Type of fault & Fault time & Residual type & $\begin{array}{c}\text { Fault flag } \\
\text { raised }\end{array}$ & $\begin{array}{c}\text { Time fault } \\
\text { Detected/isolated }\end{array}$ & Action taken & Fault type & $\begin{array}{c}\text { Time taken to } \\
\text { detect fault }\end{array}$ \\
\hline \multirow{3}{*}{$\begin{array}{l}\text { Position sensor } \\
\text { fault }\end{array}$} & \multirow{3}{*}{$42.69 \mathrm{~s}$} & Parity equation & $R_{1}$ & $42.72 \mathrm{~s}$ & \multirow{3}{*}{ Fault accommodated } & \multirow{3}{*}{ Sensor } & \multirow{3}{*}{$0.3 \mathrm{~s}$} \\
\hline & & Kalman filter & $R_{4}$ & $42.7 \mathrm{~s}$ & & & \\
\hline & & Weighted average & $w_{3}$ & $42.705 \mathrm{~s}$ & & & \\
\hline \multirow{2}{*}{$\begin{array}{c}\text { Pressure sensor } \\
\text { (n) drift }\end{array}$} & \multirow[b]{2}{*}{$20 \mathrm{~s}$} & Parity equation & $R_{3}$ & $22.47 \mathrm{~s}$ & \multirow[b]{2}{*}{ No action } & \multirow[b]{2}{*}{ Sensor } & \multirow[b]{2}{*}{$2.47 \mathrm{~s}$} \\
\hline & & Kalman filter & $R_{5}$ & $22.2 \mathrm{~s}$ & & & \\
\hline \multirow{5}{*}{ Air blockage } & \multirow{5}{*}{$35 \mathrm{~s}$} & & $R_{l}$ & $41.84 \mathrm{~s}$ & \multirow{5}{*}{ Safety mode activated } & \multirow{5}{*}{ Pneumatic } & \multirow{5}{*}{$6.84 \mathrm{~s}$} \\
\hline & & Parity equation & $R_{2}$ & $41.8 \mathrm{~s}$ & & & \\
\hline & & & $R_{3}$ & $41.8 \mathrm{~s}$ & & & \\
\hline & & \multirow[t]{2}{*}{ Kalman filter } & $R_{4}$ & $39.39 \mathrm{~s}$ & & & \\
\hline & & & $R_{5}$ & $38.64 \mathrm{~s}$ & & & \\
\hline \multirow{5}{*}{ Air leak } & \multirow{5}{*}{$18.6 \mathrm{~s}$} & & $R_{l}$ & $23.98 \mathrm{~s}$ & \multirow{5}{*}{ Safety mode activated } & \multirow{5}{*}{ Pneumatic } & \multirow{5}{*}{$5.38 \mathrm{~s}$} \\
\hline & & Parity equation & $R_{2}$ & $18.67 \mathrm{~s}$ & & & \\
\hline & & & $R_{3}$ & $18.5 \mathrm{~s}$ & & & \\
\hline & & \multirow[t]{2}{*}{ Kalman filter } & $R_{4}$ & $18.92 \mathrm{~s}$ & & & \\
\hline & & & $R_{5}$ & $18.78 \mathrm{~s}$ & & & \\
\hline \multirow{2}{*}{$\begin{array}{l}\text { Pressure sensor } \\
\text { Signal loss (p) }\end{array}$} & \multirow[b]{2}{*}{$32.95 \mathrm{~s}$} & Parity equation & $R_{2}$ & $33.2 \mathrm{~s}$ & \multirow[b]{2}{*}{ No action } & \multirow[b]{2}{*}{ Sensor } & \multirow[b]{2}{*}{$0.25 \mathrm{~s}$} \\
\hline & & Kalman filter & $R_{5}$ & $33.2 \mathrm{~s}$ & & & \\
\hline \multirow{5}{*}{ Actuator fault } & \multirow{5}{*}{$20 \mathrm{~s}$} & & $R_{l}$ & $21.55 \mathrm{~s}$ & \multirow{5}{*}{ Safety mode activated } & \multirow{5}{*}{ Pneumatic } & \multirow{5}{*}{$1.62 \mathrm{~s}$} \\
\hline & & Parity equation & $R_{2}$ & $21.62 \mathrm{~s}$ & & & \\
\hline & & & $R_{3}$ & $21.61 \mathrm{~s}$ & & & \\
\hline & & Kalman filter & $R_{4}$ & $20.65 \mathrm{~s}$ & & & \\
\hline & & & $R_{5}$ & $20.96 \mathrm{~s}$ & & & \\
\hline
\end{tabular}

